\title{
A novel adenylate isopentenyltransferase 5 regulates shoot branching via the ATTTA motif in Camellia sinensis
}

\author{
Liping Zhang ${ }^{\dagger}$, Menghan $\mathrm{Li}^{\dagger}$, Peng Yan, Jianyu Fu, Lan Zhang, Xin Li and Wenyan Han*
}

\begin{abstract}
Background: Shoot branching is one of the important agronomic traits affecting yields and quality of tea plant (Camellia sinensis). Cytokinins (CTKs) play critical roles in regulating shoot branching. However, whether and how differently alternative splicing (AS) variant of CTKs-related genes can influence shoot branching of tea plant is still not fully elucidated.

Results: In this study, five AS variants of CTK biosynthetic gene adenylate isopenteny/transferase (CSA-IPT5) with different $3^{\prime}$ untranslated region ( $3^{\prime}$ UTR) and 5' UTR from tea plant were cloned and investigated for their regulatory effects. Transient expression assays showed that there were significant negative correlations between CSA-IPT5 protein expression, mRNA expression of CSA-IPT5 AS variants and the number of ATTTA motifs, respectively. Shoot branching processes induced by exogenous 6-BA or pruning were studied, where CSA-IPT5 was demonstrated to regulate protein synthesis of CSA-IPT5, as well as the biosynthesis of trans-zeatin (tZ)- and isopentenyladenine (iP)-CTKs, through transcriptionally changing ratios of its five AS variants in these processes. Furthermore, the $3^{\prime}$ UTR AS variant 2 (3AS2) might act as the predominant AS transcript.
\end{abstract}

Conclusions: Together, our results indicate that 3AS2 of the CSA-IPT5 gene is potential in regulating shoot branching of tea plant and provides a gene resource for improving the plant-type of woody plants.

Keywords: Adenylate isopentenyltransferase, ATTTA motif, Alternative splicing (AS) variant, Axillary bud, Cytokinins (CTKs), Tea plant

\section{Background}

Shoot branching determines the aboveground architecture of a plant and lateral branches generally arise from the outgrowth of axillary buds, which is controlled by a complex interaction of phytohormones and environmental conditions [1]. The perennial tea plant (Camellia sinensis) is an economically important woody crop, with their bud leaves from lateral branches collected for tea

\footnotetext{
*Correspondence: lixin@tricaas.com; hanwy@tricaas.com ${ }^{\dagger}$ Liping Zhang and Menghan Li contributed equally to this work. Key Laboratory of Tea Quality and Safety Control, Ministry of Agriculture, Tea Research Institute, Chinese Academy of Agricultural Sciences, No. 9 Meiling South Road, Xihu District, Hangzhou 310008, Zhejiang, China
}

and food production $[2,3]$. In virture of such economic value, understanding the molecular regulatory mechanism of shoot branching in tea plant is essential [1].

Cytokinins (CTKs) are primary hormones constituting the various endogenous CTKs in plants $[4,5]$. Though mainly biosynthesized in roots, CTKs can also be locally biosynthesized to facilitate shoot [branching, in stem nodes, axillary buds and stems etc. [4, 6]. Isopentenyltransferase (IPT) catalyzes the first and rate-limiting step of CTK biosynthesis [7, 8]. The isopentenyladenine (iP)type CTKs biosynthesized in the stem node of tomato (Solanum lycopersicum) promote axillary bud sprouting and lateral branch growth [4]. Decapitation significantly induces the increase of PsIPTs expression in nodal stem 
tissues as well as CTK levels in the nodal tissue and axillary buds of pea (Pisum sativum) [3]. Adenylate IPT (A-IPT), as one of the two types of IPTs, are likely to be responsible for the bulk of CTK synthesis, including iP- and trans-zeatin ( $t$ Z)-type CTKs $[3,8,9]$. Cook et al. [10] reported that the upregulated expression of $A-I P T$ in axillary buds and stem nodes stimulates the outgrowth of axillary buds. Overexpression of the A-IPT gene in Asakurasanshoo [7], Chrysanthemum [1, 8] and Arabidopsis [8] resulted in increased branching.

Alternative splicing (AS) post-transcriptionally regulates gene expression, leading to differential mRNA stability and enriched protein diversity [11, 12]. The untranslated regions (UTRs) are generally involved in post-transcriptional regulation, which may affect mRNA stability and translation efficiency [13]. In Arabidopsis, $21.6 \%$ of AS events occur in UTRs [14]. The mRNA stability of the Arabidopsis SAUR26 gene has been conferred by polymorphisms in the $3^{\prime}$ UTR region and shown to control gene expression in response to environmental factors [13].

AU-rich elements (AREs) are sequence elements of 50-150 nt that are rich in adenosine and uridine bases and are frequently found in the $3^{\prime}$ UTRs of labile mRNAs $[15,16]$. In higher eukaryotes, the AUUUA motif, which are among the best-characterized mRNA-destabilizing determinants, and other AREs could decrease gene expression and inhibit mRNA translation (i.e., protein synthesis), at least partially by enhancing the rate of its deadenylation and subsequent mRNA degradation [16-22]. The ATTTA motif was found to be significantly enriched in transcripts with short half-lives in plants [20, $23,24]$. In the presence of three AUUUA sequences in the $3^{\prime}$ UTR, transcripts of tobacco (N. tabacum) par gene have shorter lifes [20]. Mutations in the AUUUA motifs increase mRNA stability and enhance mRNA accumulation [17, 24], whereas insertion of AUUUA motifs into a stable mRNA destabilizes the mRNA levels $[15,20]$. ATTTA and other AREs-specific stability and deadenylation of mRNA appear to be regulated by development and stress [17, 20-22]. In Arabidopsis, modulation of mRNA stability contributes to the clock-regulated expression of AtGUTs which contain AUUUA motifs [25]. In mammalian cells, many early-response genes are also regulated by instability of mRNAs containing AUUUA motifs within the $3^{\prime}$ UTR [17].

To date, the molecular mechanism that regulates shoot branching in tea plant has not been elucidated. In this study, alternatively spliced CsA-IPT5 mRNAs with different 3' UTR and 5' UTR were cloned. The relationships among the expression of CsA-IPT5 transcripts and CsA-IPT5 protein, as well as CTKs biosynthesis were examined in the shoot branching induced by exogenous
6-BA treatment and pruning, respectively. The results are expected to reveal the molecular mechanism that regulates plant shoot branching by splice variants of $C s A$ IPT5 and lay a candidate gene resources for breeding tea varieties.

\section{Results \\ Cloning of CSA-IPT gene by $3^{\prime}$ and $5^{\prime}$ rapid amplification of CDNA ends (RACE)}

The first-round $3^{\prime}$ RACE PCR at different temperatures all resulted in three gel bands, with approximate sizes of $0.9,1.0$, and $1.1 \mathrm{~kb}$, respectively (Figure S1A, lane A). The second-round $3^{\prime}$ RACE PCR obtained gel bands with similar sizes of $0.8,0.9$, and $1.0 \mathrm{~kb}$, respectively (Figure $\mathrm{S} 1 \mathrm{~A}$, lane $\mathrm{B})$. In the $5^{\prime} \mathrm{RACE}$, several gel bands were amplified at different temperatures in the first-round PCR (Figure S1B, lane A). In the second-round PCR, there was one amplified gel band at six different temperatures, approximately at $600 \mathrm{bp}$ (Figure S1B, lane B). The primers used are shown in Table S1 and S2.

\section{Sequencing analysis and multiple sequence alignment of the full-length CSA-IPT CDNA and CsA-IPT5 protein}

Based on the result of sequencing, the $5^{\prime}$ UTR and $3^{\prime}$ UTR with poly (A) were $145 \mathrm{bp}$ (1-145 bp) and $361 \mathrm{bp}$ (1187-1547 bp) in length, respectively. The full-length cDNA sequence of CsA-IPT was $1547 \mathrm{bp}$ in length, and the ORF was $1041 \mathrm{bp}$ (146-1186bp) responsible for 346 deduced amino acids. There are seven repeats of destabilizing ATTTA motifs in the full-length cDNA sequences of CSA-IPT, with four in the $5^{\prime}$ UTR, one in the ORF region, and two in the $3^{\prime}$ UTR (Figure S2). The physical positions of the ATTTA motifs are shown in Fig. 1A.

The sequence homology between the cloned CsA-IPT ORF and TEA025674.1 (TPIA, cv. Shuchazao, http://tpia. teaplant.org/index.html?tdsourcetag=s_pcqq_aiomsg) is 99.42\%. There is a lack of 67 bases in the cloned CsA-IPT compared with the $5^{\prime}$ UTR of XP_028094976.1 (NCBI, https://www.ncbi.nlm.nih.gov/). There are both additional 115 bases in the final $3^{\prime}$ UTR of the cloned CsA-IPT compared with that in the $3^{\prime}$ UTR of XP_028094976.1 and XP_028094977.1 (NCBI, https://www.ncbi.nlm.nih. gov/), respectively (Figure S3A, B). In addition, there is a lack of 186 nucleotides in the ORF sequence of the cloned CsA-IPT compared with that in the end of the CDS sequence of GWHPACFB026317 (BIG database of cv. LJ43, https://bigd.big.ac.cn/search/?dbId=gwh\&q= GWHACFB00000000), and the CDS sequence of GWHPACFB026317 was not obtained by PCR amplification in this study. The phylogenetic tree was constructed using the amino acid sequences of $15 \mathrm{~A}$-IPT protein in different plant species. Sequence alignment analysis showed that the cloned CsA-IPT had orthologs in other plant species 


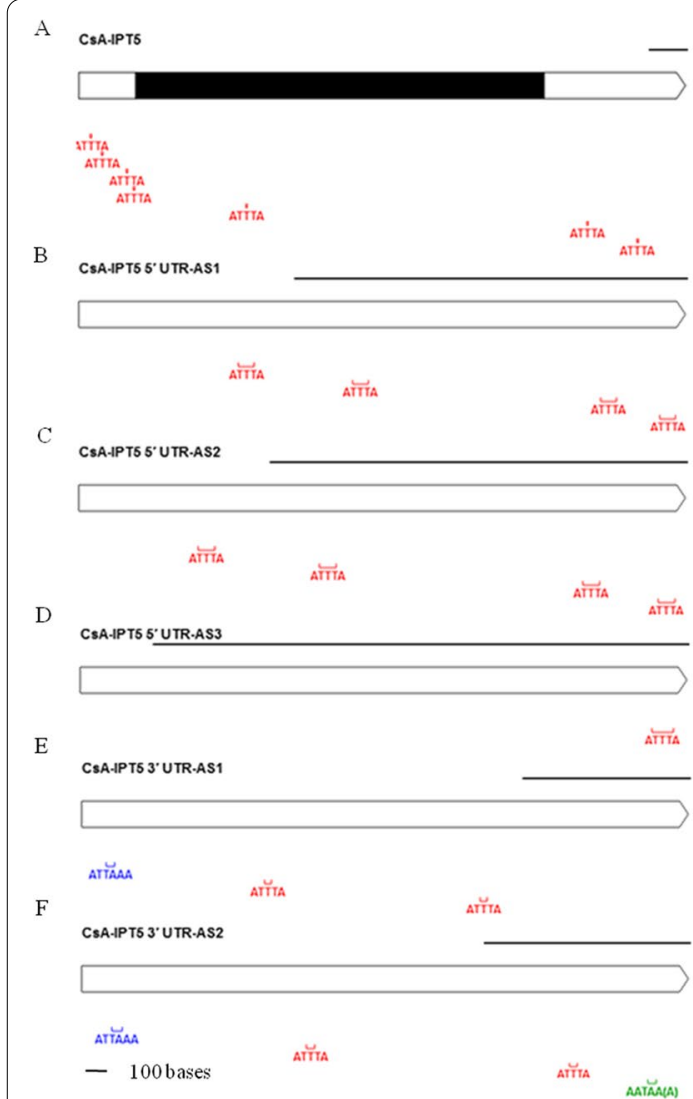

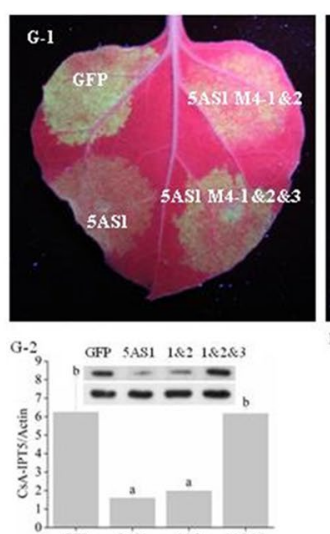
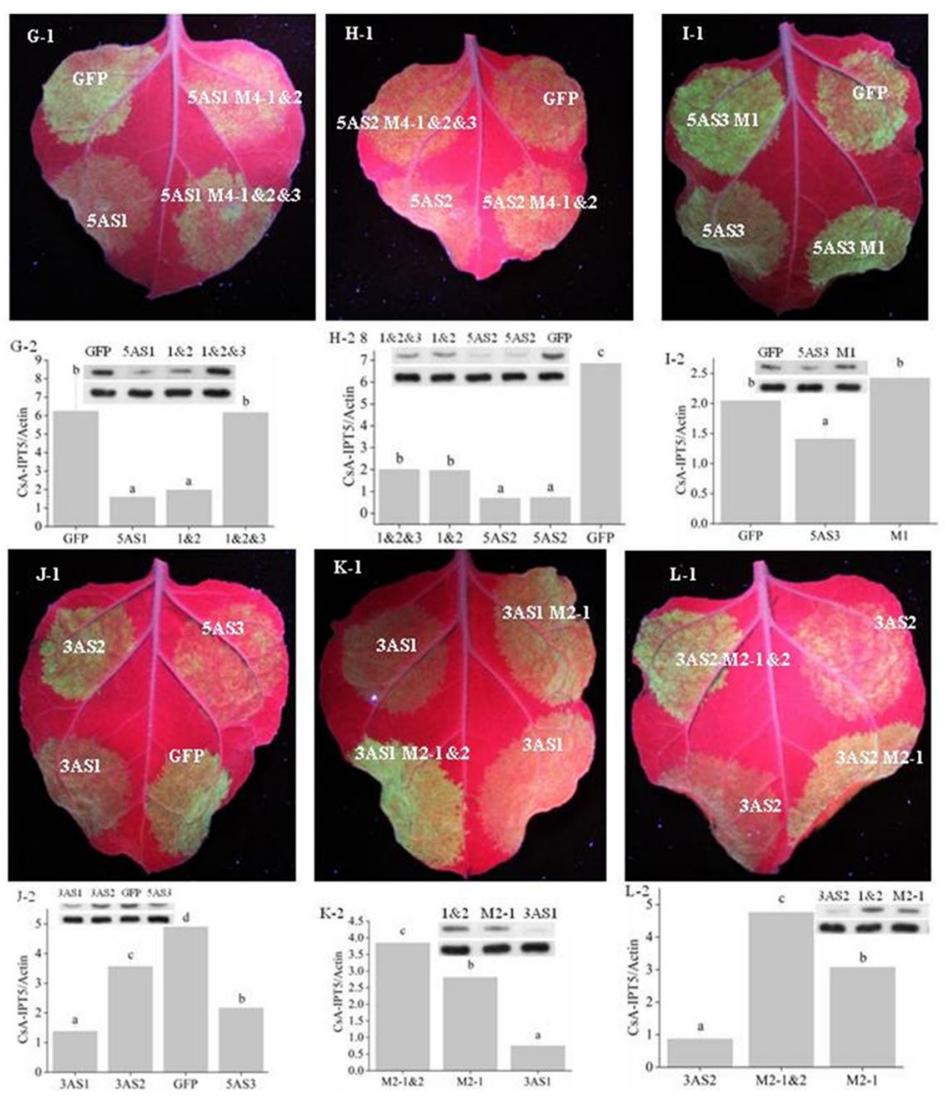

Fig. 1 Schematic diagram of the CSA-IPT5 transcripts, GFP fluorescence and qPCR detection of GFP. A-F Schematic diagram of the full-length CSA-IPT5 CDNA (A) and five CSA-IPT5 splice variants (B-F). Black rectangles represent CDS; Hollow rectangles with arrows represent UTR; a line segment represents 100 bases. The ATTTA motif and poly(A) signal sequence has been labeled with different colors, respectively. G-L GFP fluorescence and protein expression of GFP. Data are means \pm standard deviation $(S D)(n=6)$. In each figure, letters indicate significant differences among the GFP constructs $(P<0.05$, Duncan's multiple range test)

and belonged to the plant $A-I P T 5$ gene family. Thus the cloned CsA-IPT was termed as CsA-IPT5 (Figure S3C).

\section{Multiple sequence alignment of the CSA-IPT5 AS variants and subcellular localization of CsA-IPT5 protein}

Both four AS variants were found in the $5^{\prime}$ UTR and $3^{\prime}$ UTR of CsA-IPT5, respectively. Variation of ATTTA motifs in the UTRs of $5^{\prime}$ UTR AS variant (5AS) and $3^{\prime}$ UTR AS variant (3AS) mRNAs are shown in Fig. 1B-F and Figure S4. Specifically, there are four ATTTA motifs in the $5^{\prime}$ UTR of 5AS1 and 5AS2 mRNA, while there is both only one ATTTA motif in the $5^{\prime}$ UTR of $5 \mathrm{AS} 3$ and 5AS4 mRNA, respectively. Likewise, there are two ATTTA motifs in the $3^{\prime}$ UTR of 3AS1 and 3AS2 mRNA, while there is both only one ATTTA motif in the 3AS3 and 3AS4 mRNA, respectively. Furthermore, no change was found in the start or stop codon of CsA-IPT5, although AS was detected in the $5^{\prime}$ RACE and $3^{\prime}$ RACE (Figure S4), indicating the splicing variants will be translated into the same protein product-CsA-IPT5. The result of subcellular localization showed that the CsA-IPT5 protein located in the chloroplasts (Fig. 2A-F).

\section{Effect of the ATTTA motifs on CSA-IPT5 protein expression} and transcriptional activity of CSA-IPT5 transcripts in N. benthamiana leaves by multiple mutation

Both 5AS1 and 5AS2 contain four ATTTA motifs (Fig. 1B, C; Figure S5A-1, B-1). For these two, we created mutants of M4-1\&2 and M4-1\&2\&3, with the first two and the first three ATTTA motifs altered to GCCCG motifs, respectively ( $M$ represents mutant) (Figure S5A, $B)$. The result showed that the fluorescence, protein expression (Fig. 1G-J) and mRNA expression (Figure S6A-D) in the constructs were all lower than those in the relative GFP controls, except for 5AS1 M4-1\&2\&3 protein expression and 5AS3 M1 protein expression. Fluorescence was both greater from the GFP-5AS1 M4-1\&2 and GFP-5AS1 M4-1\&2\&3 clones than that from GFP$5 \mathrm{AS} 1$. The protein expression of GFP-5AS1 M4-1\&2\&3 was significantly greater than that of GFP-5AS1, however, 

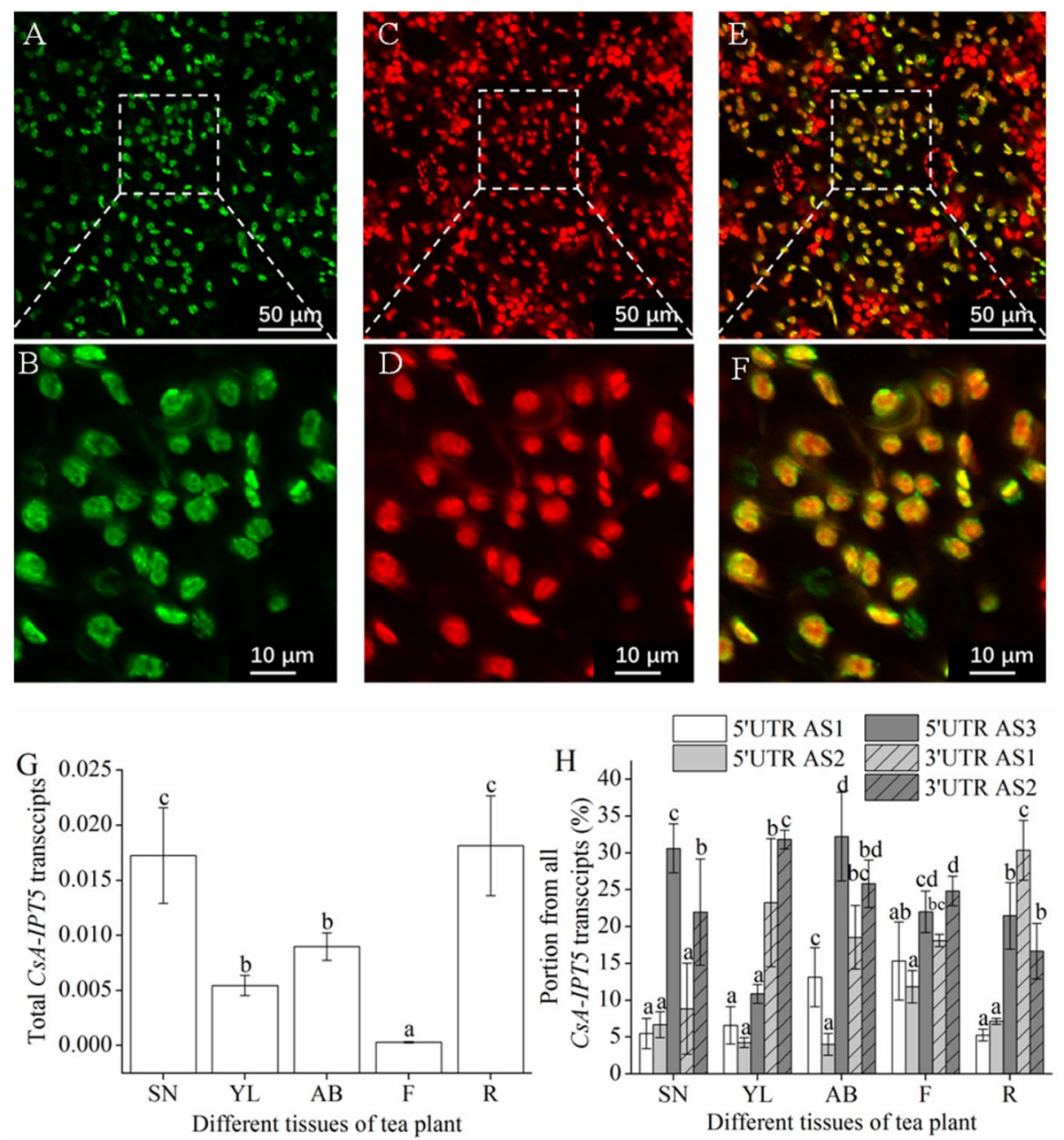

SN: Stem node; YL: Young leaf; AB: Axillary bud; F: Flower; R: Root

Fig. 2 Subcellular localization of CSA-IPT5-GFP and expression profiles of CSA-IPT5 transcripts in the different tissues of tea plant. A and $\mathbf{B}$ (magnification of A), CSA-IPT5::GFP fluorescence (green); C and D (magnification of C), chloroplast autofluorescence (red); $\mathbf{E}$ and $\mathbf{F}$ (magnification of E), merged (yellow). G, H Expression profiles of CSA-IPT5 transcripts in the different tissues of tea plant. AS patterns were expressed as ratios of the indicated splice variants to the total transcripts, and the data are mean $\pm S D(n=6)$. Letters indicate significant differences of total CSA-IPT5 among tissues $(\mathbf{G})$ and among splice variant ratios in each tissue $(\mathbf{H})(P<0.05$, Duncan's multiple range test)

there were no significant difference between that of GFP5AS1 M4-1\&2 and GFP-5AS1. Results of qPCR showed that mRNA levels of GFP-5AS1 M4-1\&2 and GFP-5AS1 M4-1\&2\&3 were about four times and 4.3 times, respectively, compared with GFP-5AS1. Moreover, the fluorescence, protein expression (Fig. 1G-1,2) and mRNA expression (Figure S6A) of GFP-5AS1 M4-1\&2\&3 was all greater than that from GFP-5AS1 M4-1\&2. On the other hand, fluorescence was both greater from the GFP-5AS2 M4-1\&2 and GFP-5AS2 M4-1\&2\&3 clones than that from GFP-5AS2 (Fig. 1H-1). The mRNA levels of GFP5AS2 M4-1\&2 and GFP-5AS2 M4-1\&2\&3 were both about twice of that resulting from GFP-5AS2, while there was no significant diffenence between that from GFP5AS2 M4-1\&2 and GFP-5AS2 M4-1\&2\&3 (Figure S6B). Moreover, the protein expression showed exactly the same pattern with the above gene expression (Fig. 1H-2). The sequence of 5AS3 contains only one ATTTA motif which was mutated into GCCCG to create the 5AS3 M1 mutant (Figure S5C; Fig. 1D). Fluorescence, protein expression (Fig. 1I-1, -2) and mRNA expression (Figure $\mathrm{S6C)}$ were all significantly greater than that from the GFP-5AS3 M1 than from GFP-5AS3.

The ATTTA motif both occurs twice in 3AS1 and 3AS2, respectively. In addition, 3AS1 and 3AS2 have one and two ATTAA(A) polyA signal sequence(s), 
respectively (Figure S5D, E; Fig. 1E, F). The fluorescence, protein expression (Fig. 1J-1, J-2), and mRNA expression (Figure S6D) in 3AS2 were significantly greater than that in 5AS3, and that in 3AS2 were significantly greater than that in 3AS1. The fluorescence, protein expression (Fig. $1 \mathrm{~K}-1,-2$ ) and mRNA levels (Figure S6E) in 3AS1 M2-1\&2 was significant greater than that in 3AS1 M2-1, and that in these two appeared to be all greater than that in the GFP-3AS1. The fluorescence, protein expression (Fig. 1L-1, -2) and mRNA levels (Figure S6F) in the 3AS2 M2-1 and 3AS2 M2-1\&2 clones were both greater than that in the GFP-3AS2, and that in GFP-3AS2 M2-1\&2 was greater than that in GFP-3AS2 M2-1.

\section{Designing and verification of the qPCR primers for CSA-IPT5 splice variants}

The primers for amplifying different AS transcripts of CsA-IPT5 were designed. Due to the presence of ASs both in the $5^{\prime}$ and $3^{\prime}$ UTRs, the qPCR primers could not be designed to detect the expression of the full-length CsA-IPT5 transcript. The corresponding qPCR primers can not be designed due to the small difference in the sequence length between 5AS3 and other splice variants and the higher AT contents in the diversity sequences. In addition, as the sequences of 5AS4 all coincide with those of the other splice variants, the amplified products of the primer pair $5 \mathrm{AS} 4 \mathrm{~F}+5 \mathrm{ASR}$ is the total expression of all the splice variants. Therefore, for the four $5^{\prime}$ UTR-AS and four $3^{\prime}$ UTR-AS obtained by RACE, the designed primers could only be used for distinguishing the expression levels of 5AS1, 5AS2, 5AS3, 3AS1, 3AS2 and the total CsAIPT5 transcript (Table S3).

The band size of the primers, including 5AS4F + 5ASR (201 bp, lane 4), 3ASF+3AS4R (199bp, lane 5), $3 \mathrm{ASF}+3 \mathrm{AS} 2 \mathrm{R} \quad(304 \mathrm{bp}, \quad$ lane 6), 3ASF + 3AS1R (427 bp, lane 7), 3ASF2+3AS1R (328bp, lane 8), and 3ASF2+3AS2R (205 bp, lane 9), all correspond to the expected size. As the primers 3ASF +3AS4R (lane 5), 3ASF + 3AS1R (lane 8) and 3ASF + 3AS2R (lane 9) have higher amplification efficiency compared to the primers 5AS4F + 5ASR (lane 4), 3ASF2 + 3AS1R (lane 7) and 3ASF2+3AS2R (lane 6), respectively, thus these three primer pairs are recommended for detcting the expression of the total transcript, 3AS1 and 3AS2 of CsA-IPT5, respectively. CsGAPDH F + CsGAPDH R1 was used for detecting the expression of housekeeping gene GAPDH (Figure S7A).

The temperature gradient amplification of different $5^{\prime}$ UTR AS was carried out. The primer pairs $5 \mathrm{AS} 1 \mathrm{~F}+5 \mathrm{ASR}, 5 \mathrm{AS} 2 \mathrm{~F}+5 \mathrm{ASR}$ and $5 \mathrm{AS} 3 \mathrm{~F}+5 \mathrm{ASR}$ were used to amplify 5AS1, 5AS2 and 5AS3, respectively. Lines $\mathrm{A} 1 \sim \mathrm{A} 6, \mathrm{~B} 1 \sim \mathrm{B} 6$ and $\mathrm{C} 1 \sim \mathrm{C} 6$ correspond to the amplified results of primer pairs 5AS1F + 5ASR (247 bp),
$5 \mathrm{AS} 2 \mathrm{~F}+5 \mathrm{ASR}$ (247bp) and 5AS3F + 5ASR (249bp) under different temperature, respectively. The primer pairs $5 \mathrm{AS} 1 \mathrm{~F}+5 \mathrm{ASR}$ and $5 \mathrm{AS} 2 \mathrm{~F}+5 \mathrm{ASR}$ both could amplify the objective band, respectively. Moreover, the primer pairs both have higher amplification efficiency under $54^{\circ} \mathrm{C}$ and $55^{\circ} \mathrm{C}$ compared with other temperature (Figure S7B).

\section{The expression of CSA-IPT5 transcripts in the different tissues of tea plant}

The expression patterns of CsA-IPT5 transcripts in the different tissues of tea plant were detected. The results showed that the CsA-IPT5 transcripts expressed in all the tested tissues. The total CsA-IPT5 transcripts in the stem node and root were the highest, followed by that in the young leaf and axillary bud. In the stem node, the 5AS3 expression/total expression (5AS3 ratio) and 3AS2 expression/total expression (3AS2 ratio) was significantly higher than the other three splice variants; in the young leaf, the 3AS1 ratio and 3AS2 ratio both were significantly higher than the other three splice variants; in the axillary bud, except for 5AS2, the other four splice variants all have relatively high expression levels, and the $5 \mathrm{AS} 3$ ratio was the highest among them; in the flower, all five splice variants have a relatively high expression level, and the 5AS1 ratio and 5AS2 ratio was relatively low compared with the three other splice variants; in the root, 5AS3, 3AS1 and 3AS2 have relatively high expression levels compared with the three other splice variants (Fig. 2G, H). Together, among the five splice variants, 5 AS3 had the highest ratio in the stem node and axillary buds, while 3AS2 showed the highest ratio in young leaf and flower, and 3AS1 appeared to be the highest in roots. Furthermore, among the five splice variants of CsA-IPT5, 3AS2 all had relatively high ratio in the five tested tissues.

\section{Involvement of CSA-IPT5 transcripts in the shoot branching induced by exogenous 6-BA}

In the internode, 6-BA induced the expression of total CsA-IPT5 transcripts, 5AS1, 5AS2, 3AS1, and 3AS2 compared with the relevant controls. Specially, in the internode, the total CsA-IPT5 transcripts induced by 6-BA were both significantly higher at $24 \mathrm{~h}$ and $6 \mathrm{~d}$ compared with the control. The 5AS1, 5AS2 and 3AS1 ratio induced by $6-\mathrm{BA}$ was all significantly higher than that in the relative control at $12 \mathrm{~h}$ and $24 \mathrm{~h}$. The $3 \mathrm{AS} 2$ ratio induced by 6-BA was significantly higher than that in the control at 6 $\mathrm{d}$ and $9 \mathrm{~d}$. However, 6-BA did not increase the 5AS3 ratio compared with the control (Fig. 3; Table S4).

In the stem node, the total CsA-IPT5 transcripts both showed a trend of first increasing and then decreasing in the control and 6-BA treatment, respectively, and 6-BA did not increase the expression of total CsA-IPT5 


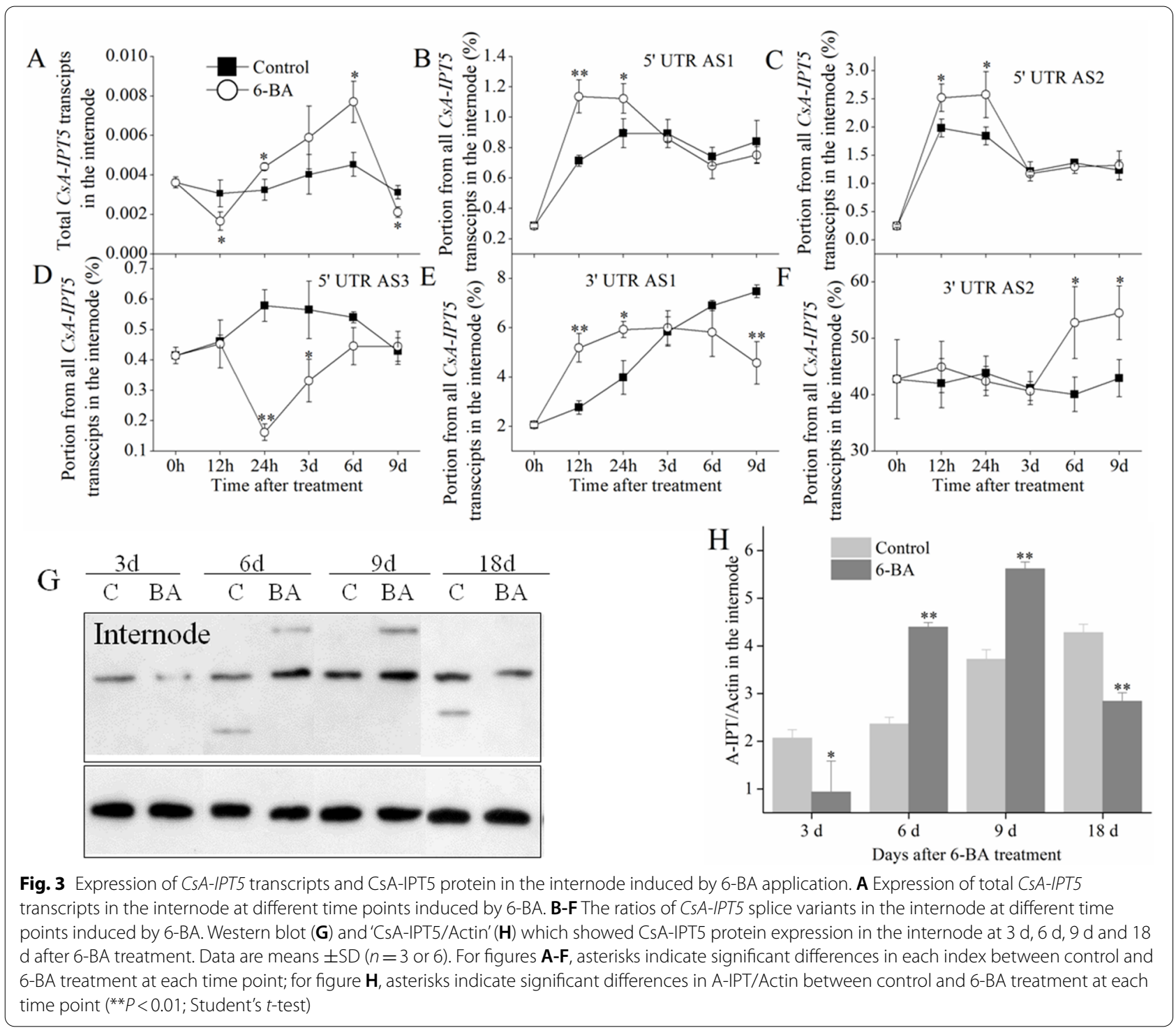

transcripts compared with the control (Fig. 4A). In the root, the expression of the total CsA-IPT5 transcripts in the 6-BA treatment was all significantly higher than that in the control at all the test time points (Fig. 4D). The iPR content both increased in the internode and stem node when treated with 6-BA (Fig. 4B, C). The protein expression of CsA-IPT5 in the internode was induced by 6-BA at $6 \mathrm{~d}$ and $9 \mathrm{~d}$ compared with the control (Fig. 3G, H), however, it did not induce the increase of CsA-IPT5 protein expression in the stem node (Fig. 4E, F).

\section{Involvement of CSA-IPT5 transcripts in regulating axillary bud development induced by pruning}

The morphology and size of the axillary buds at $16 \mathrm{~d}$ after pruning are shown in Fig. 5A. Compared with the control, pruning induced the accumulation of $t \mathrm{Z}, t \mathrm{Z}$ riboside ( $t Z R$ ) and iP riboside (iPR) in the internodes, $t Z$ and $t Z R$ in the stem node, as well as $t Z$ in the axillary buds, respectively, at $8 \mathrm{~d}$ after pruning (Fig. 5B-E). In the axillary bud, there was no change in the overall transcripts of CsA-IPT5 either in the control or in the pruning groups, and pruning did not induce the CsA-IPT5 protein expression in the axillary buds compared with the control (Fig. 5F-H).

In the stem node, the total CsA-IPT5 transcripts in the control and pruning groups both increased at first and then decreased from $0 \mathrm{~h}$ to $8 \mathrm{~d}$, and that in the the pruning groups were significantly higher than that in the control at $24 \mathrm{~h}$ and $48 \mathrm{~h}$ (Fig. 6A). The 5AS3 ratio in control showed a trend of gradual decreasing, whereas it showed 

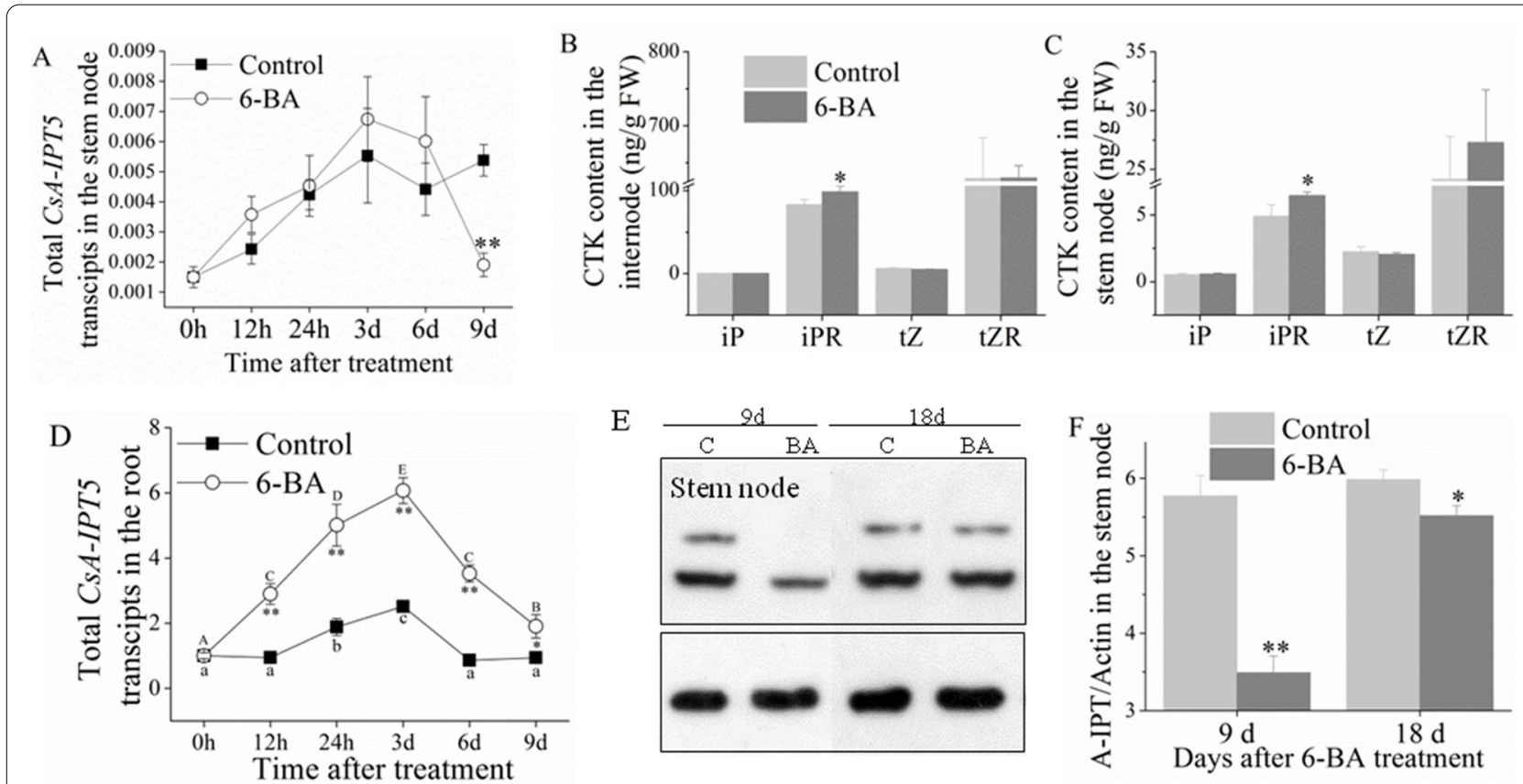

Fig. 4 Expression of total CSA-IPT5 transcripts in the stem node and root, CTKs contents in the internode and stem node, and expression of CSA-IPT5 protein in the stem node induced by 6-BA application. A,D Expression of total CSA-IPT5 transcripts in the stem node (A) and root (D) at different time points induced by 6-BA. B, C CTK contents in the internode (B) and stem node (C) induced by 6-BA. Western blot (E) and 'CSA-IPT5/ Actin' $\left.^{\prime} \mathbf{F}\right)$ which showed CSA-IPT5 protein expression in the stem node at $9 \mathrm{~d}$ and $18 \mathrm{~d}$ after 6 -BA treatment. Data are means \pm SD $(n=3$ or 6$)$. For figures $\mathbf{A}$ and $\mathbf{D}$, asterisks indicate significant differences in the expression of total CSA-IPT5 transcripts between control and 6-BA treatment at each time point; for figures $\mathbf{B}$ and $\mathbf{C}$, asterisks indicate significant differences in each index between control and 6-BA treatment; for figure $\mathbf{F}$, asterisks indicate significant differences in 'CSA-IPT5/Actin' between control and 6-BA treatment at each time point $(* * P<0.01$; Student's $t$-test)

a trend of first decreasing and then increasing after pruning in the stem node (Fig. 6D). The 3AS2 ratio in control of the stem node showed a trend of first increasing and then decreasing, whereas it showed a trend of gradual increasing in the pruning treatment (Fig. 6F). Thus, compared with the relevant control, the pruning treatment induced the expression of 5AS3 and 3AS2 respectively, in the stem node. The ratios of 5AS1, 5AS2 and 3AS1 all did not increase both in the control and pruning treatment over time (Fig. 6B, C, E). Furthermore, compared with the control, pruning induced the protein expression of CsA-IPT5 in the stem node at $8 \mathrm{~d}$ after pruning (Fig. 6G, H). Compared with the control, the expression of total CsA-IPT5 transcripts was induced by pruning in the roots but not in the internode at all the tested time points (Fig. 6I).

\section{Discussion}

Abundance of leaf buds during shoot branching is indespensible for achieving high yield and quality of tea plant, thus artificially regulating the development of the lateral branches is necessary for tea production as its obvious apical dominance [2]. A-IPTs play essential roles in the synthesis of iP- and $t$ Z-type CTKs $[9,26]$. Up to now, the function of plant $A-I P T s$ has been much studied, whereas little has been done to characterize the splice variants of plant $A-I P T$ transcripts. In the present study, clones of alternatively spliced CsA-IPT5 mRNAs of tea plant with different 3' UTR and 5' UTR were identified.

Some sequences in the UTRs, such as AU-rich elements (ARE), could modulate mRNA stability and thus regulate the transcript accumulation and translation efficiency of the mRNA $[19,27]$. Most of the reported sequences involved in mRNA stabilization reside within the $3^{\prime}$ UTR [28], meanwhile, Chen et al. [29] reported that the ATTTA-containing core domain in the $5^{\prime}$ UTR of human cytokine mRNA functions as a potent destabilizing element. The $5^{\prime}$ UTR can also be involved in modulating mRNA stability during the development and stress response of green plants [30-33]. In this study, there are one to four ATTTA motif(s) in the five $5^{\prime}$ UTR and $3^{\prime}$ UTR splice variants which were investigated for their regulatory effects.

In the current study, the biggest difference among the splice variants is in the repeats of the unstable ATTTA motifs. Our transient expression and mutation test showed differences in the repeats of the ATTTA motifs, varying from zero to four, in the fused GFP. The resuts 

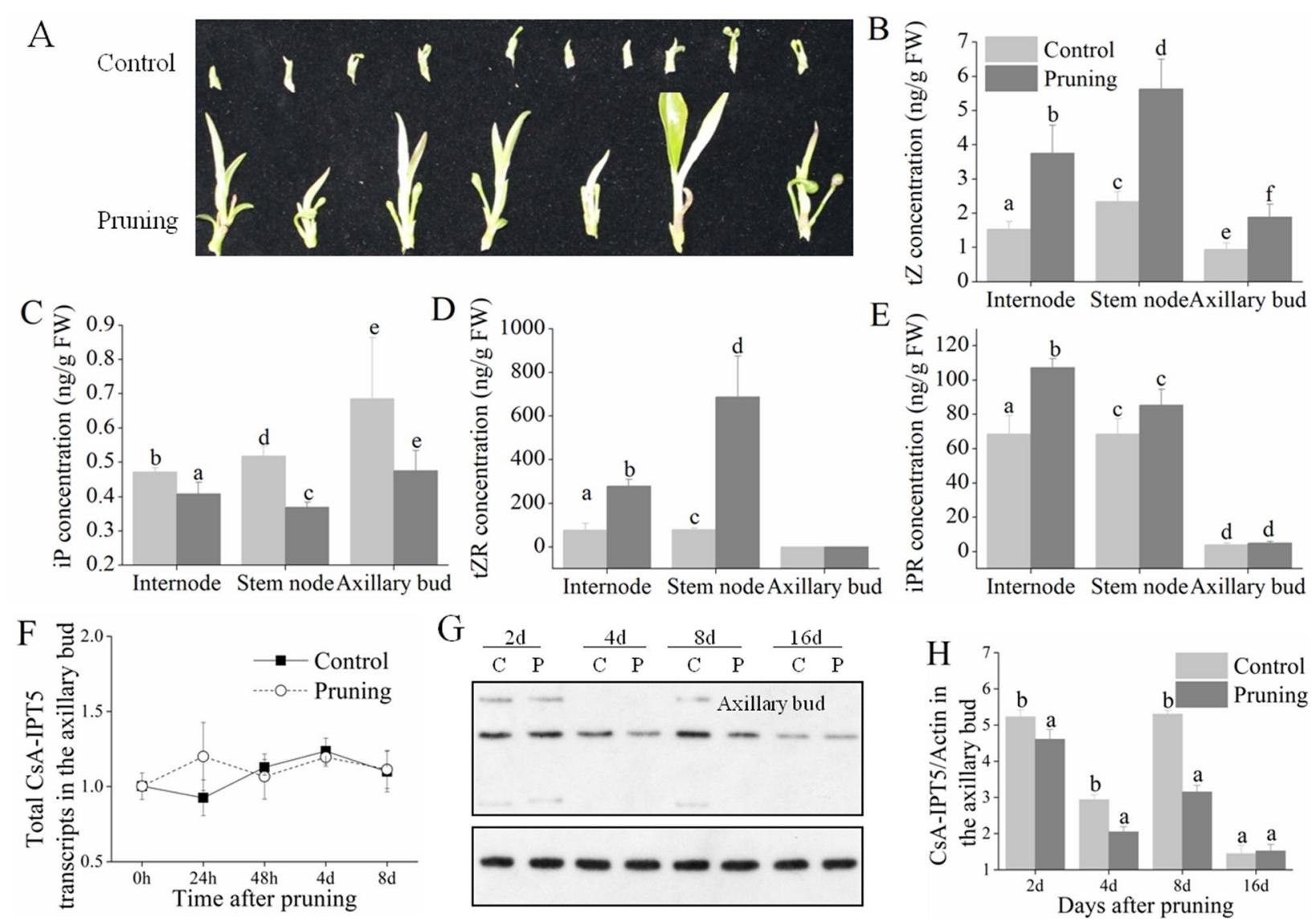

Fig. 5 The axillary bud growth vigor, CTK contents in the three tissues, and the expression of the total CSA-IPT5 transcripts and CSA-IPT5 protein in the axillary bud of the first node below the cuttings induced by the second pruning. $\mathbf{A}$ The growth vigor of the axillary bud at $16 \mathrm{~d}$ after pruning. B-E Contents of $t Z$ - and iP-type CTKs in the different tissues at $8 \mathrm{~d}$ after pruning. Data are means $\pm \operatorname{SD}(n=3)$. For each figure, letters indicate significant differences in the CTK concentration between control and pruning in each tissue $(P<0.05$, Duncan's multiple range test). $\mathbf{F}$ Expression of the total CSA-IPT5 transcripts in the axillary bud at different time points after pruning. G, H Western blot (G) and 'CsA-IPT5/Actin' (H) which showed the CSA-IPT5 protein expression levels in the axillary bud at different time points after pruning. Data are means \pm SD $(n=3)$. Letters indicate significant differences of CSA-IPT5/Actin between control and pruning at each time point $(P<0.05$, Duncan's multiple range test)

showed that except for two mutants of 5AS2 (i.e., 5AS2 M4-1\&2 and 5AS2 M4-1\&2\&3), there were all negative correlations between the transcriptional levels and the number of ATTTA motifs among the CsA-IPT5 splice variant and its relevant mutants. Furthermore, except for 5AS1 and 5AS1 M4-1\&2, as well as 5AS2 M4-1\&2 and 5AS2 M4-1\&2\&3, there were all negative correlations between the CsA-IPT5 protein expression and the number of ATTTA motifs among the AS variant and its relevant mutants. This seemed to be consistent with a number of previous studies which consider that transcripts containing a larger amount of ATTTA motifs and other ARE are more rapidly degraded than those containing a smaller amount of these motifs [17, 34]. In addition, this study showed that there were both no significant differences about the protein expression and transcriptional levels of fused GFP between that in the 5AS2
M4-1\&2 and 5AS2 M4-1\&2\&3, which have two and one ATTTA motif(s) respectively, and this is consistent with an existing report that the accumulate rates of two alternative spliced mRNAs, which with one and two ATTTA motif(s) respectively, were not distinct in vitro [24].

The poly(A) consensus signal sequence, including the AATAA(A) motif and the less frequently found ATT AAA motif, located in the 3' UTR of the mature mRNA, is essential for poly(A) tail formation during mRNA maturation [35, 36]. He et al. [24] reported that the ATT AAA sequence and AATAA sequence in the 3' UTR both upregulated plant gene expression. Here, there are two ATTTA motifs and two ATTAA(A) motifs in the 3AS2, and there is one ATTTA motif and no poly(A) signal sequence in the 5AS3. Thus, compared with 5AS3, the significantly greater fluorescence and mRNA levels of 3AS2 may be attributed to the upregulated gene 


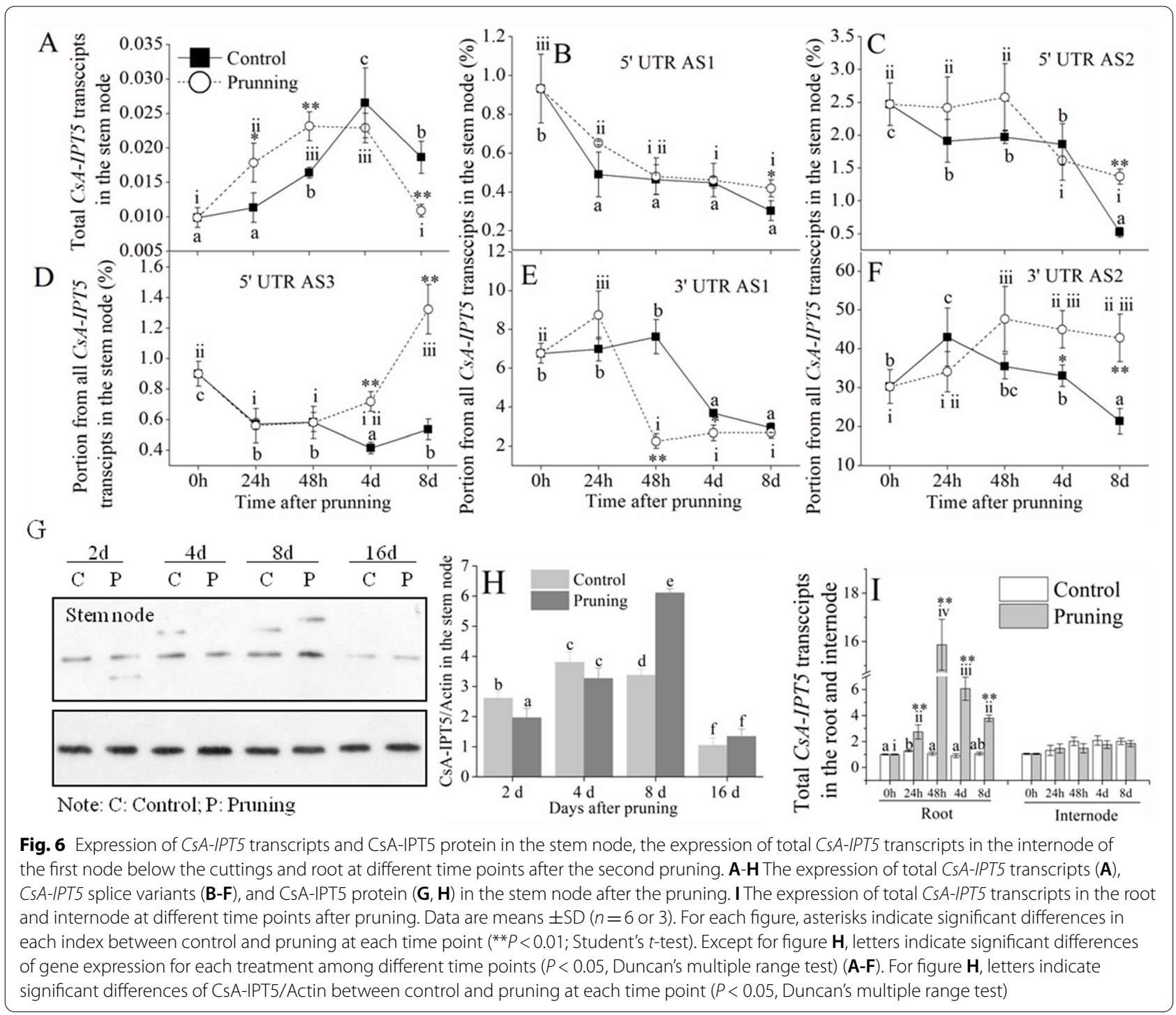

expression confered by the two AATAA(A) motifs. Likewise, compared with 3AS1, the higher expression levels of 3AS2 may be also attributed to the one more AATAA(A) motif in the 3AS2. Therefore, it is likely that the poly(A) signal sequence might be responsible for the higher transient expression levels of CsA-IPT5 splice variants, and the final transient expression level of CsAIPT5 transcripts may be the result of the interplay of the two regulatory mechanisms that can act in opposing directions.

Studies in multiple plants have indicated that each organ or tissue has its own specific AS transcripts, and tissue specific AS may have potential functions [37]. This study showed that the CsA-IPT5 splice variants are expressed in all the tested tissues, including the stem node, young leaf, axillary bud, flower, and root, and their spatial expression patterns revealed a tissue preference and differences in expression levels. Furthermore, among the five splice variants of CsA-IPT5, 3AS2 all had relatively high ratio in the five tested tissues.

Shoot branching of plants includes two stages, the formation and the subsequent elongation growth and development of axillary buds. 6-BA is a kind of synthetic cytokinin inside the plant. Our previous studies showed that 6-BA application increased the number of lateral buds 1 month after treatment, thus promoted productive lateral branch formation of tea plant [38]. The current study showed strong correlations among the increased expression of total CsA-IPT5 transcripts, 5AS1, 5AS2, 3AS1, 3AS2, increased protein expression of CsA-IPT5, and increased iPR concentrations in the internode induced by 6-BA. Nevertheless, the results indicated 6-BA did not induce the expression of CsAIPT5 transcripts and CsA-IPT5 protein in the stem node 

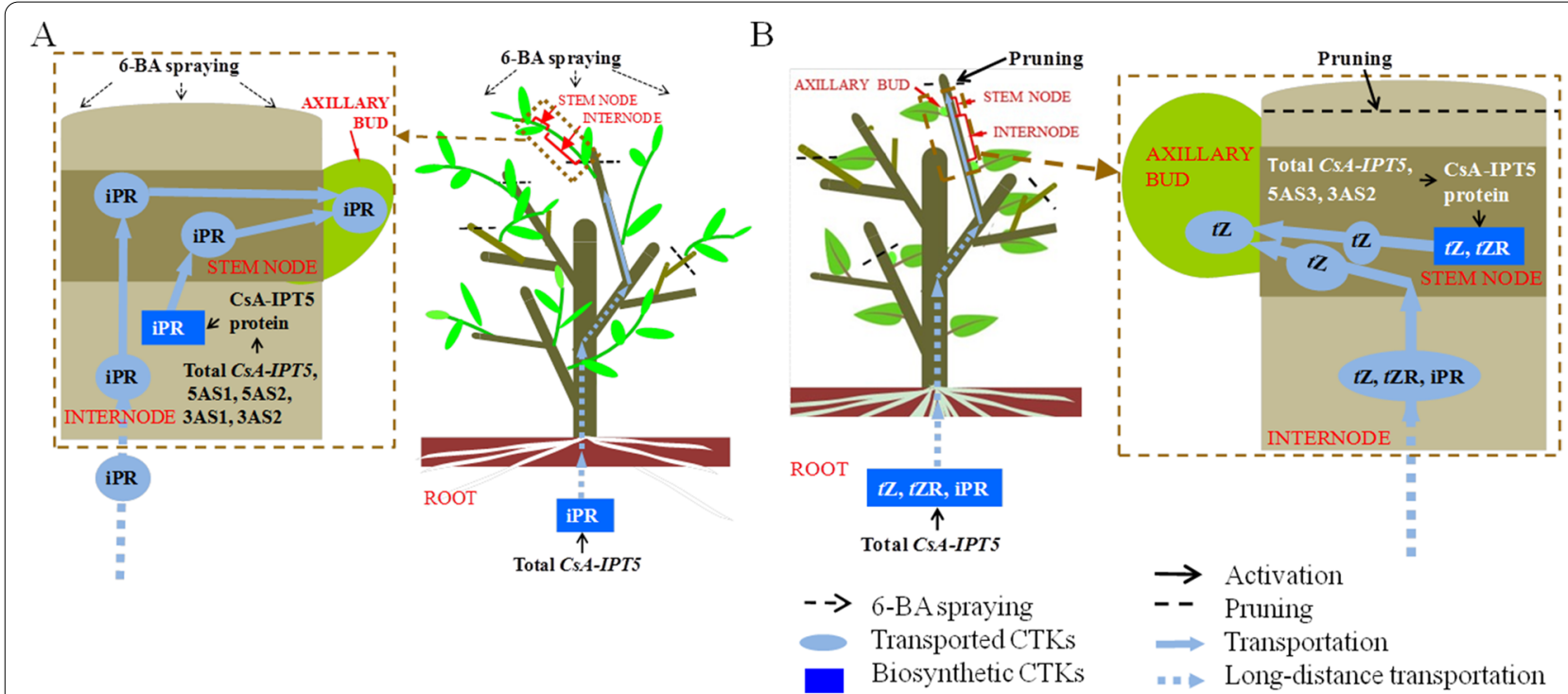

Fig. 7 Proposed models of CSA-IPT5 transcripts in the regulation of shoot branching in tea plant. A A proposed model of CSA-IPT5 transcripts in the regulation of shoot branching induced by exogenous 6-BA application in tea plant. B A proposed model of CSA-IPT5 transcripts in the regulation of shoot branching induced by the second pruning in tea plant. Each shoot branching process regulated by CSA-IPT5 transcripts are included in a single rectangle composed by brown dashed lines, respectively. Black dashed arrows represent exogenous 6-BA spraying. Black dashed lines represent the second pruning. Light blue ellipses represent the transported CTKs. Blue rectangles represent locally biosynthetic CTKs. Black arrows represent activation. Light blue solid arrows represent transportation, whereas light blue dashed arrows represent long-distance transportation.

compared with the control. Thus, 6-BA may induce iPR biosynthesis in the internode, meanwhile, the increased iPR concentration detected in the internode might also be transported from root, as 6-BA increased the expression of total CsA-IPT5 in the root compared with the control. Thus, the increased iPR concentration detected in the stem node might be transported from the internode and root. Together, it can be speculated that after 6-BA treatment, the iPR biosynthsized in the internode and root was transported to the axillary bud and promote its development (Fig. 7a). Furthermore, the 5AS1, 5AS2, 3AS1 and 3AS2 all played key roles in the shoot branching induced by 6-BA.

Pruning is an essential agronomic practice in tea cultivation $[39,40]$. In this study, the increased expression of total CsA-IPT5 transcripts, 5AS3 and 3AS2, increased protein expression of CsA-IPT5, as well as increased concentrations of $t Z$ and $t Z R$ in the stem node induced by pruning treatment were shown to be positively correlated. Thus the increased concentrations of $t \mathrm{Z}$ and $t \mathrm{ZR}$ detected in the stem node at least partly biosynthsized by itself. Furthermore, pruning induced the expression of total CsA-IPT5 transcripts in the root but not in the internode. Thus, it can be speculated that the increased concentrations of $t Z, t Z R$ and $\mathrm{PPR}$ in the internode tested here may come from root. However, as pruning did not induce the expression of total CsA-IPT5 transcripts and CsA-IPT5 protein in the axillary bud, it is possible that the increased $t Z$ accumulation in the developing axillary bud is transported from other tissues, including the stem node and root, and promoted the axillary bud developement (Fig. 7b). Furthermore, in the axillary bud development induced by pruning, the 5AS3 and 3AS2 both played key roles.

The regulatory effects of ATTTA motif and other ARE during different developmental stages or under environmental stimui have been frequently reported in higher eukaryotes. For example, optimal metabolic adaptation to oxygen variations relies on ARE-mediated mRNA stability and thus the regulation of gene transcription and enzyme activity in Drosophila cells [22]. The splice variants of high-glucose-regulated (HGRG-14) mRNA, which contains different numbers of ATTTA motifs, is produced and correlates well with HGRG-14 protein levels under different glucose conditions in human mesangial cells [19]. As discussed above, it is most likely that during shoot branching induced by 6-BA treatment or pruning, the expression patterns of the five UTR splice variants of $C s A-I P T 5$ were differently regulated in this study. The splice variants regulate the synthesis of CsA-IPT5 protein as well as tZ- and iP- CTKs through changing the ratio of the five mRNAs. Furthermore, the 3AS2 might act as the most important transcript. 


\section{Conclusions}

In summary, the present study examined the molecular mechanisms of shoot branching regulated by the AS transcripts of CsA-IPT5 in tea plant. The five CsA-IPT5 splice variants might play roles through compensation or competition with each other. Of the five, the 3AS2 of CsA-IPT5 may be the predominant transcript and act as a key determinant with specific functions in the dynamic equilibrium. This study highlights the underlying ASbased regulatory mechanisms in tea plant shoot branching, and it provides a material (gene) for improving the plant-type of woody plants.

\section{Methods}

\section{Plant material and growth conditions}

Adult tea plants (C. sinensis L.) of the widely grown cultivars in China, Longjing 43 (LJ43) and Zhongcha 108 (ZC108), were used in this study. All experiments were conducted in the tea garden of the Tea Research Institute, Chinese Academy of Agricultural Sciences, Hangzhou, Zhejiang province, China. No permissions were necessary to collect such samples.

\section{Isolation of the full-length CDNA of CsA-IPT}

In the preliminary experiments, due to the lack of genomic data of tea plant cv. LJ43, we obtained the fulllength cDNA of CsA-IPT in C. sinensis cv. LJ43 using RACE technology. The total RNA was extracted from the tea leaves of cv. LJ43 using the modified CTAB method. The first-strand cDNA for $5^{\prime} \mathrm{RACE}$ and $3^{\prime} \mathrm{RACE}$ of $C s A$ IPT was synthesized using Tiosbio SUPERSWITCH ${ }^{\mathrm{TM}}$ PCR cDNA synthetic kit (ST0091, Baoying Tonghui Biotechnology Co., Ltd., Beijing, China), respectively. Purified PCR products were cloned into the $\mathrm{pMD} 18-\mathrm{T}$ vector ( $p E A S Y$-T5 Zero Cloning Kit, TransGen Biotech, Beijing, China), then transformed into the Trans1-T1 phage resistant chemically competent cell (TransGen Biotech). DNA sequencing was performed by TsingKe (Hangzhou, China). The verified forward primer of real-time quantitative PCR (qPCR) (CsIPT5-F query_L1) and the $3^{\prime}$ adaptor primers (3AP) provided in the RACE kit were used for the 3' RACE-PCR at first, and then performed by nested PCR using the primers CsIPT5-3R2 and 3AP. The first amplification of the $5^{\prime}$ RACE was performed using the primers CsIPT5-5R1 and 5AP and then performed by nested PCR using the primers CsIPT5-5R2 and 5AP in the kit. The primers and the primer combinations used for RACE PCR are shown in Tables S1 and S2. The gradient program for RACE PCR was: $94^{\circ} \mathrm{C}$ for $5 \mathrm{~min}$, followed by 35 or 31 cycles of $94^{\circ} \mathrm{C}$ for $30 \mathrm{~s}, 50-68^{\circ} \mathrm{C}$ for $30 \mathrm{~s}$, and $72^{\circ} \mathrm{C}$ for $3.5 \mathrm{~min}$, and then a $10 \mathrm{~min}$ final extension at $72{ }^{\circ} \mathrm{C}$.
Bioinformatics analysis of the cloned CSA-IPT gene The CsA-IPT5 cDNA sequence, deduced amino acid sequence, and open reading frame (ORF) were analyzed online or by the corresponding bioinformatics software. The deduced CsA-IPT5 and other known plant A-IPT genes were aligned with online CLUSTALW (http://www. ebi.ac.uk/Tools/msa/clustalw2).

\section{Subcellular localization of CsA-IPT5}

The primers for amplifying the CsA-IPT5 cDNA fulllength ORF without the stop codon was designed (forward primer: 5'-CgACgACAAgACCgTcaccATGAGA ATTTCATTCTCACCT-3'; reverse primer: 5'-GAG GAGAAGAGCCGTCGCCGAGTTGTGGCGGCGACA $\left.-3^{\prime}\right)$, and the ORF sequence was fused to the green fluorescence protein (GFP, accession number U87973). The fusion gene was inserted into the plant binary expression vector $p J P 186$ that contains the GFP, yielding the transformation vector 35S: $A$-IPT-GFP. This vector was used for the transient transformation of Nicotiana tabacum leaves. The epidermal cells of $N$. tabacum leaves were observed for subcellular localization of CsA-IPT5 using a confocal laser scanning microscope (Nikon A1, Japan). GFP fluorescence was excited at $488 \mathrm{~nm}$ and detected using a 492-548 $\mathrm{nm}$ emission filter. Chlorophyll autofluorescence was excited at $543 \mathrm{~nm}$ and detected using a 620$700 \mathrm{~nm}$ emission filter.

\section{Transient expression of CsA-IPT5 splice variants specific GFP of $5^{\prime}$ and $3^{\prime}$ UTR in transfected $\boldsymbol{N}$. benthamiana}

Different repeats of ATTTA elements within the splice variants in $5^{\prime}$ and $3^{\prime}$ UTR of CsA-IPT5 were mutated to GCCCG by oligonucleotide-directed mutagenesis using the altered sites mutagenesis Kit (Promega, Madison, WI, USA) [27]. The splice variants and the mutated genes, as well as the corresponding sequences, are shown in Figure S5. For agroinfiltration, Nicotiana benthamiana plants were maintained in growth chambers at $20-25^{\circ} \mathrm{C}$ throughout the assays. Agrobacterium tumefacient infiltration was performed as described by He et al. [24]. Bacterial cells were harvested and re-suspended in infiltration buffer to a final $\mathrm{OD}_{600}=1.0$. In co-infiltration experiments, equal volumes of each suspension were mixed before infiltration. GFP fluorescence was observed under a long-wavelength UV light (Black Ray model B 100A, Ultra-Violet Products Ltd., Cambridge, United Kingdom). The comparable constructs and the pCV1300:GFP control were infiltrated into a single leaf to reduce the background difference of the tobacco leaves. 


\section{Real-time quantitative PCR (qPCR)}

Total RNA was isolated using an RNA extraction kit (Tiangen Biotech, Beijing, China) and reverse transcribed using the PrimeScriptTM RT-PCR Kit (TaKaRa, Otsu, Japan), following the manufacturer's instructions. The qPCR assay was performed on an ABI 7500 Real-Time PCR system (Applied Biosystems, Foster City, CA, USA) using a SYBR Green PCR Master Mix (Takara, Shiga, Japan). The qPCR cycling conditions were as follows: $95^{\circ} \mathrm{C}$ for $30 \mathrm{~s}$ and 40 cycles of $95^{\circ} \mathrm{C}$ for $5 \mathrm{~s}$. CsGAPDH was chosen as a stable reference gene for expression normalization. Relative gene expression of the CsAIPT5 transcripts was calculated by the $2^{-\triangle \mathrm{CT}}$ method, and the expression ratio of each splice variant (i.e., expression of splice variant/total expression) was calculated. All qPCR detection of shoot branching experiments were carried out using six independent biological replicates.

The GFP transcript in transfected tobacco leaves were detected in qPCR using the GFP forward primer (TGT CAGTGGAGAGGGTGAA) and reverse primer (CGT CCTTGAAGAAGATGGTC). The PCR reaction was denatured at $95^{\circ} \mathrm{C}$ for $1 \mathrm{~min}$, followed by 45 cycles of $15 \mathrm{~s}$ at $95^{\circ} \mathrm{C}, 20 \mathrm{~s}$ at $55^{\circ} \mathrm{C}$, and $20 \mathrm{~s}$ at $72^{\circ} \mathrm{C}$. The N. benthamiana Ubiquitin $\mathrm{C}$ gene (accession Number: AB026056.1) was chosen as the reference gene for qPCR analysis. Relative gene expression of the GFP was calculated by the $2^{-\triangle \triangle \mathrm{CT}}$ method.

\section{Designing and verification of the qPCR primers for CSAIPT5 splice variants}

Total RNA was extracted using the CTAB method, and genomic DNA was removed. RNA reverse transcription was carried out with HiScript II Q RT SuperMix for qPCR (+gDNA wiper) R223-01 (Vazyme, Nanjing, China). Thirteen primers were designed according to the 5'and 3' UTRs of the CsA-IPT5 mRNA. The primers and the PCR amplification program can be seen in Table S3. The PCR program was: $94^{\circ} \mathrm{C}$ for $5 \mathrm{~min}$, followed by 35 cycles of $94^{\circ} \mathrm{C}$ for $30 \mathrm{~s}, 59^{\circ} \mathrm{C}$ for $30 \mathrm{~s}$, and $72^{\circ} \mathrm{C}$ for $30 \mathrm{~s}$, and then a $10 \mathrm{~min}$ extension at $72{ }^{\circ} \mathrm{C}$.

\section{Production of the CsA-IPT5-specific polyclonal antibody and western blot analysis}

Young adult New Zealand white rabbits were selected. The first antigen concentration was $1 \mathrm{mg} / \mathrm{ml}, 0.5 \mathrm{ml} /$ rabbit, two-free - four free antigen concentration in half, the same dose. The first immunization was made with complete adjuvant. The injections two to four were made with incomplete adjuvant. The collected serum was tested using ELISA. The purified antibodies were detected using ELISA and western blot, respectively. An anti-CsA-IPT5 peptide antibody was purified with antigen affinity purification (Hua An Bioscience Technology, Hangzhou, China).

Total protein of the $N$. benthamiana leaves and tea plant tissues was extracted using a plant protein extraction kit containing a protease inhibitor cocktail. The GFP protein level in $N$. benthamiana leaves was measured by Western Blotting. The first antibody GFP (Abcam, ab183734) and the goat anti-Rabbit IgG(H+L) secondary antibody (Thermo Pierce, 31,210) were used here. The synthetic GFP constructs were listed in Figure S5.

The total protein of tea plant tissues was quantified using the BCA protein assay kit. For western-blot analysis, the extracted proteins were separated by $10 \%$ SDSPAGE and transferred to PVDF membranes. The primary antibody (anti-CsA-IPT5 peptide antibody) and secondary antibody were hybridized successively. The signal was detected using the SuperSignal ${ }^{\circledR}$ West Dura Extended Duration Substrate.

\section{6-BA treatment and the pruning treatment}

For shrub-type mature tea garden in which only spring tea was picked up, tea plants usually need pruning twice a year. For the first pruning, tea trees were heavily pruned after spring tea in late April, and all the branch leaves that are approximately $50 \mathrm{~cm}$ above the ground are pruned; the second pruning was carried out around July 20.

The 6-BA treatment of tea plant was described in our previous report [38]. Specifically, mature tea plants of cv. LJ43 that were heavily pruned in late April were used. Working solutions of $200 \mathrm{mg} \mathrm{L}^{-1}$ 6-benzyladine (6-BA) (J\&K Scientific Ltd., Beijing, China) were prepared, and $0.1 \%(\mathrm{v} / \mathrm{v})$ Tween 20 was added before spraying. When the apical buds and two to three leaves below the bud grew out, the foliar portion of tea bushes was sprayed with 6-BA solutions. Control tea bushes were simultaneously sprayed with distilled $\mathrm{H}_{2} \mathrm{O}$ containing the same ratio of Tween 20 .

For the second pruning experiment, the mature tea plants cv. ZC108 which were heavily pruned in late April were used. The second pruning was conducted at a height that was $20 \mathrm{~cm}$ higher than the last cut surface on July 25, 2019. Control tea bushes were not pruned. Three tissues of the first node below the cuttings, including the internode, stem node and axillary bud, were sampled at $0 \mathrm{~h}$, $12 \mathrm{~h}, 24 \mathrm{~h}, 36 \mathrm{~h}, 48 \mathrm{~h}, 4 \mathrm{~d}, 8 \mathrm{~d}$, and $16 \mathrm{~d}$ after pruning.

For the studies of 6-BA and pruning treatments, the samples both were collected from more than 10 trees as one biological replicate, frozen in liquid $\mathrm{N}_{2}$ immediately after sampling, and stored at $-80^{\circ} \mathrm{C}$ until analysis. 


\section{Measurement of iP- and tZ-type CTK contents}

The detection of iP- and $t Z$-type CTK contents was carried out using Agilent 1290 Infinity LC Ultra Performance Liquid Chromatography (UHPLC, Agilent) and 5500 QTRAP mass spectrograph (MS, AB SCIEX) (UHPLC-MS) (Shanghai Applied Protein Technology Co. Ltd., China).

\section{Statistical analysis}

Data were analyzed using Statistica (SAS Institute Inc., http://www.statsoft.com). Data were analyzed by oneway ANOVA; if the ANOVA analysis was significant $(P<0.05)$, Duncan's multiple range test was used to detect the significant differences between/among groups. Differences between the control and treatment were determined by Student's $t$-tests.

\section{Supplementary Information}

The online version contains supplementary material available at https://doi. org/10.1186/s12870-021-03254-5.

Additional file 1: Figure S1. The $3^{\prime}$ RACE and 5' RACE PCR amplification of tea plant CSA-IPT. Figure S2. Analysis of the ORF and regulatory elements of the full-length CDNA of CSA-IPT. Figure S3. Multiple sequence alignment of the nucleotide sequences among the cloned full-length CSAIPT CDNA in cv. Longjing 43 and the reported CSA-IPT5 in cv. Shuchazao, and phylogenetic tree construction of A-IPT in several plant species. Figure S4. Alignment of $5^{\prime}$ RACE and $3^{\prime}$ RACE AS of tea plant CSA-IPT5, respectively. Figure $\mathbf{S 5}$. The nucleotide sequences and specific motifs in the GFP fluorescence of CSA-IPT5 UTR splice variants. Figure S6. The qPCR detection of GFP. Data are means \pm standard deviation (SD) $(n=6)$. In each figure, letters indicate significant differences among the GFP constructs $(P<0.05$, Duncan's multiple range test). Figure S7. Amplification of the different primer pairs for the $3^{\prime}$ UTR-AS and $5^{\prime}$ UTR-AS of CSA-IPT5.

Additional file 2: Table S1. The RACE PCR primers for CSA-IPT gene. Table S2. Primer combination used for RACE PCR of CSA-IPT gene. Table S3. Primer sequences used for real-time quantitative PCR ( $\mathrm{PPCR}$ ) of CSA-IPT5 AS variants and housekeeping gene of tea plant. Table S4. The total CSA-IPT5 transcipts and the expression ratio of each splice variant in the internode induced by 6-BA application.

Additional file 3.

Additional file 4.

\section{Acknowledgements}

Not applicable.

\section{Authors' contributions}

HWY, LX and ZLP planned and designed the research. LMH, ZLP, YP, FJY and $Z L$ performed the experiments and analyzed data. ZP and LX wrote the manuscript. ZLP and LMH contributed equally. All authors read and approved the final manuscript.

\section{Funding}

This work was supported by Key Project of International Science \& Technology Cooperation,

National Key Research and Development Programme of China

(2017YFE0107500), Zhejiang Province Public Welfare Technology Application Research Project (LGN21 C020005), and Innovation Project of the Chinese Academy of Agricultural Sciences (CAAS-ASTIP-2015-TRICAAS). The funders. provided the financial support to the research.

\section{Availability of data and materials}

All the data and materials that are required to reproduce these findings can be shared by contacting the first author, zhanglp2016@tricaas.com (ZLP).

\section{Declarations}

Ethics approval and consent to participate

Not applicable.

\section{Consent for publication}

Not applicable.

\section{Competing interests}

The authors declare that they have no competing interests.

Received: 4 March 2021 Accepted: 23 September 2021

Published online: 09 November 2021

\section{References}

1. Tan M, Li G, Qi S, Liu X, Chen X, Ma J, et al. Identification and expression analysis of the IPT and CKX gene families during axillary bud outgrowth in apple (Malus domestica Borkh.). Gene. 2018;651:106-17.

2. Zhang Q, Cai M, Yu X, Wang L, Guo C, Ming R, et al. Transcriptome dynamics of Camellia sinensis in response to continuous salinity and drought stress. Tree Genet Genomes. 2017;13:78.

3. Zhu J, Wang X, Xu Q, Zhao S, Tai Y, Wei C, et al. Global dissection of alternative splicing uncovers transcriptional diversity in tissues and associates with the flavonoid pathway in tea plant (Camellia sinensis). BMC Plant Biol. 2018;18:266.

4. Chen X-J, Xia X-J, Guo X, Zhou Y-H, Shi K, Zhou J, et al. Apoplastic $\mathrm{H}_{2} \mathrm{O}_{2}$ plays a critical role in axillary bud outgrowth by altering auxin and cytokinin homeostasis in tomato plants. New Phytol. 2016;211:1266-78.

5. Waldie T, Leyser O. Cytokinin targets auxin transport to promote shoot branching. Plant Physiol. 2018;177:803-18.

6. Dun EA, Germain AS, Rameau C, Beveridge CA. Antagonistic action of strigolactone and cytokinin in bud outgrowth control. Plant Physiol. 2012;158:487-98.

7. Zeng XF, Zhao DG. Expression of IPT in Asakura-sanshoo (Zanthoxylum piperitum (L.) DC. f. inerme Makino) alters tree architecture, delays leaf senescence, and changes leaf essential oil composition. Plant Mol Biol Rep. 2016;34:649-58.

8. Ishak A, Dong L, Rong H, Zhang S, Zhao L. Isolation and functional analysis of the regulation of branching by isopentenyl transferase gene CMIPT1 in Chrysanthemum morifolium cv. 'Jinba'. Am J Mol Biol. 2018;8:92-101.

9. Miyawaki K, Tarkowski P, Matsumoto-Kitano M, Kato T, Sato S, Tarkowska $D$, et al. Roles of Arabidopsis ATP/ADP isopentenyltransferases and tRNA isopentenyltransferases in cytokinin biosynthesis. P Natl Acad Sci USA. 2006;103:16598-603.

10. Cook NC, Bellstedt DU, Jacobs G. Endogenous cytokinin distribution patterns at budburst in granny Smith and Braeburn apple shoots in relation to bud growth. Sci Hortic. 2001;87:8753-63.

11. Srivastava AK, Lu Y, Zinta G, Lang Z, Zhu JK. UTR-dependent control of gene expression in plants. Trends Plant Sci. 2018;23:248-59.

12. Zuo Y, Feng F, Qi W, Song R. Dek42 encodes an RNA-binding protein that affects alternative pre-mRNA splicing and maize kernel development. J Integr Plant Biol. 2019;61:728-48.

13. Wang Z, Yang L, Wu D, Zhang N, Hua J. Polymorphisms in cis-elements confer SAUR26 gene expression difference for thermo-response natural variation in Arabidopsis. New Phytol. 2020. https://doi.org/10.1111/nph. 17078.

14. Reddy ASN, Marquez Y, Kalyna M, Barta A. Complexity of the alternative splicing landscape in plant. Plant Cell. 2013;25:3567-683.

15. Feldbrügge M, Arizti P, Sullivan ML, Zamore PD, Belasco JG, Green PJ. Comparative analysis of the plant mRNA-destabilizing element, DST, in mammalian and tobacco cells. Plant Mol Biol. 2002;49:215-23.

16. Barreau C, Paillard L, Osborne HB. AU-rich elements and associated factors: are there unifying principles? Nucleic Acids Res. 2005;33:7138-50. 
17. Voeltz GK, Steitz JA. AUUUA sequences direct mRNA deadenylation uncoupled from decay during Xenopus early development. Mol Cell Biol. 1998;18:7537-45.

18. Zhu S, Li W, Zeng X, Jiang D, Mao X, Liu H. Molecular cloning and sequencing of two 'short chain' and two 'long chain' $\mathrm{K}\left({ }^{+}\right)$channel-blocking peptides from the Chinese scorpion Buthus martensii Karsch. FEBS Lett. 1999;457:509-14.

19. Hyodo S, Kawakoshi A, Bartolo RC, Takei Y, Toop T, Donald JA. Extremely high conservation in the untranslated region as well as the coding region of CNP mRNAs throughout elasmobranch species. Gen Comp Endocr. 2006;148:181-6.

20. Ohme-Takagi M, Taylor CB, Newman TC, Green PJ. The effect of sequences with high AU content on mRNA stability in tobacco. P Natl Acad Sci USA. 1993:90:11811-5.

21. von Roretz C, Marco SD, Mazroui R, Gallouzi IE. Turnover of AU-richcontaining mRNAs during stress: a matter of survival. Wires RNA. 2011;2:336-47.

22. de Toeuf B, Soin R, Nazih A, Dragojevic M, Jurènas D, Delacourt N, et al. ARE-mediated decay controls gene expression and cellular metabolism upon oxygen variations. Sci Rep. 2018;8:5211.

23. Narsai R, Howell KA, Millar AH, O'Toole N, Small I, Whelan J. Genomewide analysis of mRNA decay rates and their determinants in Arabidopsis thaliana. Plant Cell. 2007;19:3418-36.

24. He Q, Peng J, Yan F, Lin L, Lu Y, Zheng H, et al. Intron retention and $3^{\prime}$-UTR analysis of Arabidopsis dicer-like 2 transcripts. Mol Biol Rep. 2012;39:3271-80.

25. Gutiérrez RA, Ewing RM, Cherry JM, Green PJ. Identification of unstable transcripts in Arabidopsis by cDNA microarray analysis: rapid decay is associated with a group of touch- and specific clock-controlled genes. Proc Natl Acad Sci U S A. 2002;99:11513-8.

26. Wang X, Lin S, Liu D, Gan L, Li Y. Evolution and roles of cytokinin genes in angiosperms 1: do ancient IPTs play housekeeping while non-ancient IPTS play regulatory roles? Hortic Res. 2020;7:28.

27. Vincenti MP, Coon Cl, Lee O, Brinckerhoff CE. Regulation of collagenase gene expression by IL-1 beta requires transcriptional and post-transcriptional mechanisms. Nucleic Acids Res. 1994;22:4818-27.

28. Sajjanar B, Deb R, Raina SK, Pawar S, Brahmane MP, Nirmale AV, et al. Untranslated regions (UTRs) orchestrate translation reprogramming in cellular stress responses. J Therm Biol. 2017;65:69-75.

29. Chen CY, Gherzi R, Ong SE, Chan EL, Raijmakers R, Pruijn GJM, et al. AU binding proteins recruit the exosome to degrade ARE-containing mRNAs. Cell. 2001;107:451-64.
30. Hua XJ, Cotte BVD, Montagu MV, Verbruggen N. The $5^{\prime}$ untranslated region of the At-P5R gene is involved in both transcriptional and posttranscriptional regulation. Plant J. 2001;26:157-69.

31. Hansen ER, Petracek ME, Dickey LF, Thompson WF. The $5^{\prime}$ end of the pea ferredoxin-1 mRNA mediates rapid and reversible light-directed changes in translation in tobacco. Plant Physiol. 2001;125:770-8.

32. Bhat $S$, Tang $L$, Krueger $A D$, Smith $C L$, Ford SR, Dickey LF, et al. The Fed-1 $(\mathrm{CAUU})_{4}$ element is a $5^{\prime}$ UTR dark-responsive mRNA instability element that functions independently of dark-induced polyribosome dissociation. Plant Mol Biol. 2004;56:761-73.

33. Hulzink RJM, de Groot PFM, Croes AF, Quaedvlieg W, Twell D, Wullems GJ, et al. The $5^{\prime}$-untranslated region of the $n t p 303$ gene strongly enhances translation during pollen tube growth, but not during pollen maturation. Plant Physiol. 2002;129:342-53.

34. Gingerich TJ, Feige JJ, LaMarre J. AU-rich elements and the control of gene expression through regulated mRNA stability. Anim Health Res Rev. 2004;5:49-63.

35. Edwalds-Gilbert G, Veraldi KL, Milcarek C. Alternative poly(a) site selection in complex transcription units: means to an end? Nucleic Acids Res. 1997;25:2547-61.

36. Matoulkova E, Michalova E, Vojtesek B, Hrstka R. The role of the $3^{\prime}$ untranslated region in post-transcriptional regulation of protein expression in mammalian cells. RNA Biol. 2012;9:563-76.

37. Jiao Y, Tausta SL, Gandotra N, Sun N, Liu T, Clay NK, et al. A transcriptome atlas of rice cell types uncovers cellular, functional and developmental hierarchies. Nat Genet. 2009:41:258-63.

38. Zhang LP, Shen C, Wei JP, Han WY. Effects of exogenous 6-benzyladenine on dwarfing, shoot branching, and yield of tea plant (Camellia sinensis). Hortscience. 2018;53:651-5.

39. Kumar R, Bisen JS, Singh M, Bera B. Effect of pruning and skiffing on growth and productivity of Darjeeling tea (Camellia sinensis L.). Int J Tech Res Appl. 2015;3:28-34.

40. Sun M, Zhang C, Lu M, Gan N, Chen Z, Deng W-W, et al. Metabolic flux enhancement and transcriptomic analysis displayed the changes of catechins following long-term pruning in tea trees (Camellia sinensis). J Agr Food Chem. 2018;66:8566-73.

\section{Publisher's Note}

Springer Nature remains neutral with regard to jurisdictional claims in published maps and institutional affiliations.
Ready to submit your research? Choose BMC and benefit from:

- fast, convenient online submission

- thorough peer review by experienced researchers in your field

- rapid publication on acceptance

- support for research data, including large and complex data types

- gold Open Access which fosters wider collaboration and increased citations

- maximum visibility for your research: over $100 \mathrm{M}$ website views per year

At BMC, research is always in progress.

Learn more biomedcentral.com/submissions 